\title{
Sistema de gestión del conocimiento para la seguridad del paciente $y$ análisis de eventos adversos en IPS, profesionales independientes y transporte asistencial
}

\author{
Edison Alonso Sosa* \\ María Elizabeth Duque Montes ${ }^{* *}$ \\ Lina Paola Pasaje Beltrán ***
}

Recibido: 4 de julio de 2020

Revisado: 16 de julio de 2020

Aprobado: 23 de julio de 2020

Citar como:

Sosa, E. A., Duque Montes, M. E. y Pasaje Beltrán, L. P. (2021). Sistema de gestión del conocimiento para la seguridad del paciente y análisis de eventos adversos en IPS, profesionales independientes y transporte asistencial. Revista CIFE, 23(38).

https://doi.org/10.15332/22484914.6134

\section{Resumen}

El sistema de salud para seguridad del paciente ha evolucionado desde la aplicación de métodos manuales hasta la descripción en bases de datos de las herramientas de apoyo, sin embargo, aún no se cuenta con un sistema de gestión inteligente que facilite la respuesta oportuna a los pacientes. Por lo anterior, esta investigación desarrolló un sistema de gestión del conocimiento para la seguridad del paciente, con el fin de dar solución a los principales problemas en la calidad y atención en la salud brindada por parte de las Entidades Administradoras de Planes de Beneficios (EAPB), Instituciones Prestadoras de Servicios de Salud (IPS), Servicios de Transporte Especial de Pacientes y profesionales independientes, así mismo, se busca generar la interacción de dicha información con las entidades departamentales, distritales y municipales de salud, el Instituto Nacional de Salud y el Organismo Técnico de Administración de la Cuenta de Alto Costo. Para tal efecto, se realizó la caracterización de la situación actual a través del método de descripción cuantitativa tecnológico, con base en una descripción en cascada, promoviendo el uso de tecnologías digitales en salud, ampliando los sistemas de información sanitaria y recopilando datos de estricta vigilancia. De esta manera, se espera facilitar la notificación y el análisis de riesgos, eventos adversos y otros indicadores de obligatorio cumplimiento, así como el reporte ante los entes territoriales de salud en

\footnotetext{
* Universidad del Rosario, Administración en Salud. ORCID: https://orcid.org/0000-0002-8028-6832

** Universidad del Rosario, Administración en Salud. ORCID: https://orcid.org/0000-0001-8704-7677

${ }^{* * *}$ Universidad del Rosario, Administración en Salud. ORCID: https://orcid.org/0000-0002-4855-0584
} 


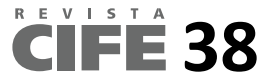

Colombia lo que contribuiría a la implementación y el mejoramiento de prácticas asistenciales seguras, a través del seguimiento de los indicadores de gestión de seguridad y calidad.

Palabras claves: seguridad, paciente, salud pública, EAPB, eventos adversos, notificación.

Clasificación JEL: L10, L26, L23.

\section{Knowledge management system for patient safety and analysis of adverse events in Health Service Provider Institutions, independent professionals and patient transport}

\begin{abstract}
The health system for patient safety has evolved from the application of manual methods to the description in databases of support tools, however there is still no intelligent management system that facilitates the timely response to patients. Therefore, this research developed a knowledge management system for patient safety in order to solve the main problems in the quality and health care provided by Benefit Plan Administration Entities (EAPB, in Spanish), Health Service Provider Institutions (IPS, in Spanish), Special Patient Transport Services, independent professionals, as well as to generate interaction of such information with departmental, district and municipal health entities, the National Health Institute and Technical Agency for the Administration of the High Cost Account. For this purpose, the current situation was characterized through the technological quantitative description method based on a cascade description, promoting the use of digital health technologies, expanding health information systems, and collecting strict surveillance data. In this way, it is expected to facilitate the notification and analysis of risks, adverse events and other mandatory indicators, as well as the reporting to territorial health entities in Colombia, contributing to the implementation and improvement of safe health care practices through the monitoring of safety and quality management indicators.
\end{abstract}

Keywords: safety, patient, public health, EAPB, adverse events, notification.

JEL Classification System: L10, L26, L23.

\section{Introducción}

La seguridad del paciente es una responsabilidad de todas las naciones del mundo, por consiguiente deben establecerse bases científicas para mitigar los eventos adversos asociados a la atención en salud y mejorar la calidad en la prestación de los servicios de salud (Organización Mundial de la Salud, 2002).

Cada año, millones de pacientes sufren lesiones o mueren a causa de una atención sanitaria poco segura y de mala calidad. Muchas prácticas médicas y riesgos relacionados con la atención sanitaria se están convirtiendo en problemas importantes para la seguridad de los 
pacientes y contribuyen significativamente a la carga de daños por atención poco segura (Organización Mundial de la Salud, 2019).

Las Instituciones Prestadoras de Servicios de Salud (IPS), los profesionales independientes y las empresas de transporte asistencial, son los encargados de garantizar una atención segura y de calidad a cada uno de los usuarios que demandan dichos servicios; por tal razón, cada institución debe crear y ejecutar planes estratégicos de seguridad que se conviertan en factores críticos para el éxito organizacional, ofreciendo mecanismos para cerrar la brecha que existe entre lo que la organización es capaz de realizar y lo que verdaderamente necesita hacer en beneficio de la calidad y seguridad de los pacientes (Summers, 2006).

Llevar un control de indicadores claves de desempeño; permite mantener un enfoque de mejora continua a largo plazo; tomar decisiones con base en hechos y encontrar soluciones, no fallas, claves en la gestión de la seguridad del paciente y el análisis de eventos adversos. Esto significa que tanto el personal asistencial como administrativo están involucrados en el proceso de crear y mantener una organización eficiente y segura (Summers, 2006).

A continuación, se muestran hechos que evidencian la importancia de garantizar una gestión de seguridad en el paciente y la importancia del análisis de eventos adversos para su prevención y control.

Los errores de medicación son una de las principales causas de lesiones y daños evitables en los sistemas de atención sanitaria, de hecho, se estima que en todo el mundo el costo asociado a los errores de medicación asciende a US 42000 millones de dólares anuales (Atiken \& Gorokhovich, 2012).

Las infecciones relacionadas con la atención sanitaria afectan 7 y 10 de cada 100 pacientes hospitalizados en países de ingresos altos y países de ingresos bajos y medios, respectivamente (World Health Organization, 2011).

Los procedimientos quirúrgicos poco seguros provocan complicaciones en hasta el $25 \%$ de los pacientes. Anualmente, casi siete millones de pacientes quirúrgicos sufren complicaciones significativas, de los que un millón fallece durante la intervención o inmediatamente después de ella (Chang, 2011).

Las prácticas de inyección poco seguras en entornos de atención sanitaria pueden transmitir infecciones, entre ellas el VIH y los virus de la hepatitis B y C, lo que conlleva un peligro directo para los pacientes y los profesionales sanitarios. Estás prácticas representan una carga de daños estimada en 9,2 millones de años de vida perdidos por discapacidad y muerte en todo el mundo (Jha et al., 2013).

Los errores diagnósticos afectan aproximadamente al $5 \%$ de los adultos que reciben atención ambulatoria, y más de la mitad de estos errores pueden llegar a causar daños graves es más, 
la mayoría de las personas se ven afectadas por algún error de diagnóstico a lo largo de su vida (Singh et al., 2014).

Las prácticas de transfusión poco seguras exponen a los pacientes al riesgo de reacciones adversas y la transmisión de infecciones. Los datos sobre reacciones adversas a la transfusión procedentes de un grupo de 21 países muestran una incidencia media de 8,7 reacciones graves por cada 100000 unidades de componentes sanguíneos distribuidas (Janssen \& Behr-Gross, 2014).

Las tromboembolias venosas son una de las causas más frecuentes y prevenibles de daños a los pacientes, estás suponen una tercera parte de las complicaciones atribuidas a la hospitalización. Se estima que anualmente se registran 3,9 millones de casos en los países de ingresos altos y 6 millones de casos en los países de ingresos bajos y medios (Raskob et al., 2014).

Todos estos hechos muestran la importancia para las IPS de poder contar con un sistema de gestión del conocimiento que les permita monitorear sus estándares de seguridad y analizar sus eventos adversos.

La septuagésima segunda asamblea de la Organización Mundial de la Salud (OMS) establece promover el uso de las nuevas tecnologías, especialmente el de las tecnologías digitales para la salud, incluso para desarrollar y ampliar los sistemas de información sanitaria y para prestar apoyo a la recopilación de datos para la vigilancia y la notificación de riesgos, eventos adversos y otros indicadores de daño en distintos niveles de los servicios sanitarios y sociosanitarios; a la par, se vela por la protección de los datos personales y se fomenta el uso de soluciones digitales para mejorar la seguridad de la atención sanitaria (World Health Organization, 2005).

De acuerdo con lo anterior, el objetivo de esta investigación se centró en el desarrollo de un sistema de gestión del conocimiento para apoyar la mejora en la toma de decisiones, a partir de los indicadores de gestión de la seguridad del paciente y el análisis de eventos adversos ocurridos en el ámbito del trabajo, IPS, profesionales independientes y empresas de transporte asistencial, facilitando la articulación en la evaluación y seguimiento llevada a cabo por parte de los entes de control. Labor que se inicia con el diseño del sistema de información, el cual brindó una metodología administrativa a los entes mencionados, para el análisis de eventos adversos e implementación de prácticas seguras en los programas de seguridad de paciente y mejoramiento de la calidad.

Este sistema de gestión del conocimiento resulta de suma importancia, principalmente porque permite evidenciar la trazabilidad de los factores críticos de éxito (indicadores de seguridad de paciente) y establecer planes de mejoramientos por medio de la ruta crítica, a través de las prácticas asistenciales seguras establecidas en los paquetes instruccionales del Ministerio de 
Salud en Colombia, como el respectivo análisis de los eventos adversos por medio de la metodología del protocolo de Londres y sus respectivas subclasificaciones.

Los sistemas de información ofrecen beneficios económicos por medio de su eficiencia, la gestión de datos y, fundamentalmente la administración del cuidado de los usuarios (Fátima y Colomo-Palacios, 2018). La función principal de los sistemas de información hospitalarios consiste en la recolección de información, su almacenamiento y procesamiento, la contabilidad financiera; el análisis de información de gestión ejecutiva el análisis de decisiones de información estadística; y la comunicación de datos (Wei, 2011). Además, se encargan de analizar los altos costos resultantes de los errores cometidos en la prescripción de medicamentos; errores que han sido minimizados con la ayuda de un software al momento de la prescripción de los medicamentos (Borges et al., 2011).

Esta investigación se divide en 6 secciones: se componen, en primer lugar, de un resumen ejecutivo, donde se refleja la metodología, los resultados y las conclusiones más importantes de esta investigación. En segundo lugar, de una introducción, donde se indica la importancia que tiene el desarrollo de un software de seguridad del paciente, para el reporte, la evaluación, seguimiento y control de este tipo de eventos generados durante la atención en salud. En tercer lugar, de un marco teórico, que sustenta el desarrollo de dicho sistema de información, describiendo su implementación, ventajas, normatividad vigente, etc. Esto fundamentado en investigaciones previas y actuales sobre el tema; lo que fue llevado a cabo mediante la revisión sistemática de literatura, obtenida de bases como: Pubmed, Cinhal, Scopus, CRAI (Centro de Recursos para el Aprendizaje y la Investigación, de la Universidad del Rosario) y Google Scholar, entre otras. En cuarto lugar, de una exposición del método de estudio utilizado, en el que se brinda información detallada del paso a paso de cómo surge y se materializa el software de seguridad del paciente. En quinto lugar, de los resultados obtenidos; y finalmente, en sexto lugar, de las conclusiones de esta investigación.

\section{Método}

El sistema de gestión del conocimiento para el abordaje de la seguridad del paciente y el análisis de eventos adversos permite que las IPS, profesionales independientes y empresas de transporte especial integren a sus procesos misionales una metodología de mejoramiento continuo, por medio de la adopción y evaluación de prácticas seguras, establecidas en los paquetes instruccionales y el análisis de eventos adversos e incidentes que se presentan durante la prestación de los servicios de salud, de acuerdo con los lineamientos del Ministerio de Salud de Colombia.

Su funcionamiento está encaminado, especialmente, a recopilar la información asociada a los eventos adversos e incidentes que se presentan en el desarrollo de las actividades propias de los entes mencionados, como su gestión desarrolla e incrementa las prácticas asistenciales 
seguras - por medio de autoevaluaciones anuales y de acuerdo con el costo asociado a la no adopción de dichas prácticas-, cuál es el riesgo que asumiría cualquiera de estos entes de no tomar acciones encaminadas a su mitigación cuántos serían los usuarios afectados por esto.

Tanto la gestión como la recopilación de la información, constituyen un instrumento que permite llevar la trazabilidad organizacional, con base en indicadores de gestión y el mejoramiento de los procesos asistenciales, al crear barreras y defensas para la mitigación de los eventos adversos y otros incidentes con el fin de disponer de la información necesaria para la consulta y toma de decisiones.

\section{Ideas de diseño}

De acuerdo con lo establecido en la Resolución 3100 de 2019, más específicamente en el estándar de los procesos prioritarios asistenciales, los entes en cuestión deben desarrollar prácticas seguras y contar con la evidencia del desarrollo de estas, de forma documentada o sistematizada, para los siguientes procesos asistenciales:

- Asegurar la correcta identificación del paciente en los procesos asistenciales.

- Gestionar y desarrollar una adecuada comunicación entre las personas que atienden y cuidan a los pacientes, lo que debe incluir enfoques diferenciales.

- Detectar prevenir y reducir infecciones asociadas con la atención en salud.

- Garantizar la funcionalidad de los procedimientos de consentimiento informado.

- Mejorar la seguridad en la utilización de medicamentos.

- Prevenir y reducir la frecuencia de caídas.

- Garantizar la atención segura de la gestante y el recién nacido.

- Prevenir complicaciones asociadas a disponibilidad y manejo de sangre, componentes y transfusión sanguínea.

- Prevenir úlceras por presión.

- Mejorar la seguridad en los procedimientos quirúrgicos.

- Detectar, analizar y gestionar eventos adversos.

Se debe realizar el análisis y gestión de los eventos adversos e incidentes que se presenten en el desarrollo de las labores de cada ente, asimismo, debe ser reportado de forma semestral ante las instituciones encargadas del control de estas actividades en Colombia.

Por lo anterior, el personal asistencial necesita una plataforma que le permita realizar el registro en tiempo real de los eventos adversos e incidentes que se presentan en el transcurso de sus actividades asistenciales, lo que puede llevarse a cabo a través de internet, por medio 
de cualquier dispositivo móvil o de escritorio Por otra parte, los referentes de seguridad necesitan centralizar dicha información para desarrollar el respectivo análisis, llevar a efecto las gestiones pertinentes implementarlas prácticas seguras asistenciales Los principales propósitos del sistema de gestión para el abordaje de la seguridad del paciente y el análisis de eventos adversos, giran en torno a centralizar la información de las diferentes dependencias de las instituciones prestadoras de servicios de salud, compartir entre las mismas la gestión de los eventos adversos e incidentes, con el propósito de su mitigación, y, por último, obtener información útil para el desarrollo de las prácticas asistenciales seguras.

Este proyecto propone un sistema de información bajo la tecnología de red privada virtual (VPN), que puede conectar fácilmente a cualquiera de los entes objeto de estudio e inclusive a las instituciones de vigilancia y control, por medio de un acceso local o externo. Los usuarios de la intranet local se conectan por este medio y los usuarios remotos acceden por internet a la intranet, mediante el uso de la tecnología VPN, de este modo se realiza la interconexión de la información y se posibilita su consulta.

El almacenamiento de datos, necesario para analizar las prácticas seguras y en llevar a efecto el análisis y gestión de incidentes y eventos adversos, se realizará por medio de una tecnología de sincronización en tiempo real en una base de datos SQL Server El sistema está conformado por un BackEnd, que está desarrollado en un lenguaje de programación C\#.net, y un FrontEnd, desarrollado en un lenguaje de programación Google Angular 9 El mecanismo de conexión de la base de datos, el BackEnd, el FrontEnd y la tecnología VPN se da por medio de un Web Service, a través del protocolo https y mediante lenguaje XML, en un servidor empresarial exclusivo para las IPS, los profesionales independientes y empresas de transporte especial.

De otro lado, la aplicación debe cumplir con unos estándares mínimos de acuerdo con la normatividad actual; de igual forma, debe permitir el análisis grafico de los indicadores y su parametrización, según los servicios prestados por cada ente. Ya que, de acuerdo con la complejidad de las labores realizadas por cada uno, se aplicarán algunas o todas las prácticas seguras de los paquetes instruccionales.

\section{Resultado}

Para evidenciar el uso y aplicación de la herramienta como elemento clave en la toma de decisiones, principalmente en lo referente al seguimiento de los indicadores de gestión de la seguridad del paciente, se registrará la información del año 2019 de una IPS de transporte especial. Los paquetes instruccionales que aplican a este tipo de ente están relacionados con: asegurar la correcta identificación de los usuarios que demandan los servicios de salud; detectar prevenir y reducir las infecciones asociadas con la atención en salud; mejorar la 
seguridad en la utilización de medicamentos; y el desarrollo de procesos para la prevención y reducción en la frecuencia de caídas.

A continuación, se muestra el módulo asociado al paquete instruccional denominado "Asegurar la correcta identificación de los usuarios que demandan los servicios de salud", módulo que evalúa la proporción de incidentes o eventos adversos asociados a fallas en la identificación de los usuarios. Cabe anotar que la metodología es idéntica y se aplica para los diferentes paquetes instruccionales expuestos anteriormente. La figura 1 muestra los resultados del análisis de este módulo, comprendidos entre los meses de enero y diciembre de 2019.

Figura 1. Incidentes o eventos adversos asociados a la identificación de los usuarios

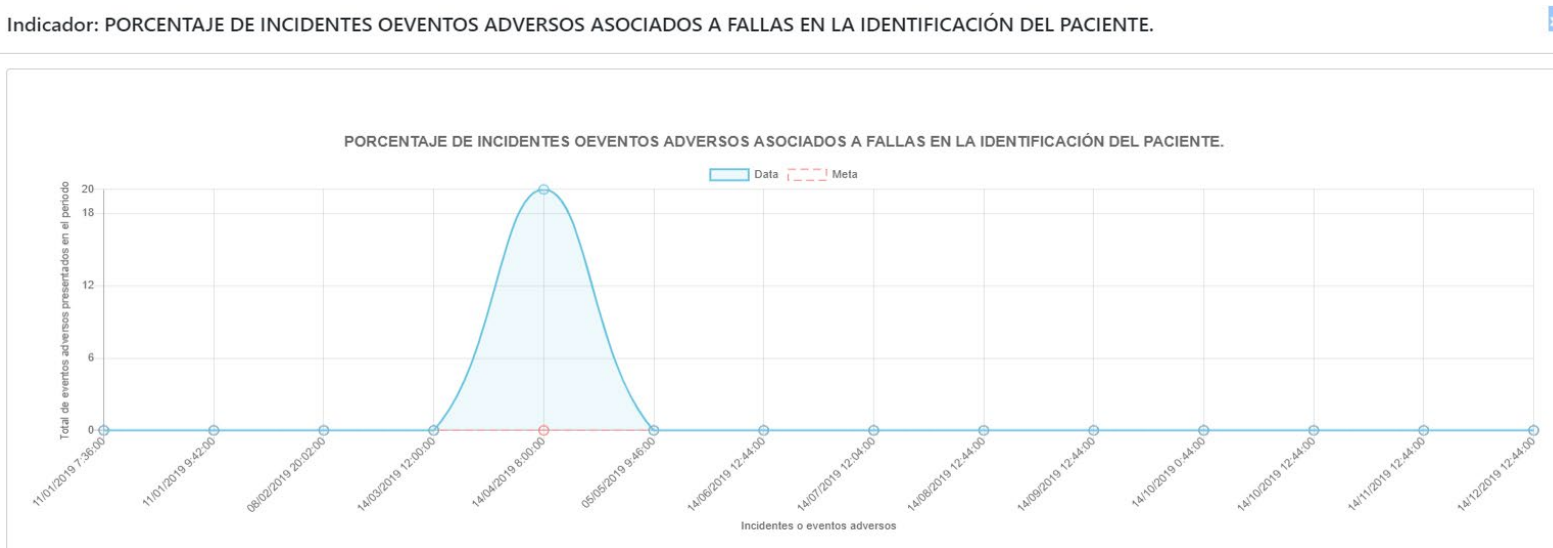

Los resultados evidencian que durante el mes de abril se presentó un incremento del $20 \%$ de los incidentes asociados a la correcta identificación de los usuarios que demandan los servicios de salud — su meta es que sea de $0 \%$ - Frente a esto, se adoptaron prácticas seguras para la mitigación de dichos eventos, las cuales fueron registradas en la herramienta y priorizadas por medio de la matriz costo, riesgo y volumen, las más relevantes o con mayor oportunidad de mejoramiento son:

- Diseñar e implementar una eficaz entrevista de salud al paciente al momento de su ingresa a la institución, para la adecuada recolección de la información

- Contar con los equipos necesarios para la recolección eficiente de los datos del paciente.

- Elaborar formatos de historia clínica que contengan los datos personales necesarios e indispensables para lograr una identificación precisa. (ver figura 2). 


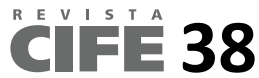

Figura 2. Prácticas seguras para la mitigación de eventos asociados a la identificación de usuarios

\begin{tabular}{|c|c|c|c|c|c|c|}
\hline Proceso Asistencial & Falla Activa & Practica Segura & Oportunidad de Mejora & Calidad Esperada & Medicion Inicial & \\
\hline $\begin{array}{l}\text { ASEGURAR LA } \\
\text { CORREATA } \\
\text { IDENTIFICACIÓN DEL } \\
\text { PACIENTE EN LOS } \\
\text { PROCESOS } \\
\text { ASISTENCIALES }\end{array}$ & $\begin{array}{l}\text { Falla de identificación al } \\
\text { ingreso del paciente. }\end{array}$ & $\begin{array}{l}\text { Realizar una adecuada entrevista inicial al paciente en el } \\
\text { momento del ingreso a la institución, para la adecuada } \\
\text { recolección de la información. }\end{array}$ & $\begin{array}{l}\text { Establecer un protocolo } \\
\text { institucional con las preguntas } \\
\text { necesarias para una adecuada } \\
\text { recolección de la información. }\end{array}$ & $\begin{array}{l}\text { El } 100 \% \text { del personal asistencial } \\
\text { aplica la entrevista inicial para una } \\
\text { recolección adecuada de } \\
\text { información. }\end{array}$ & $\begin{array}{l}\text { El } 72 \% \text { del personal asistencial } \\
\text { aplica la entrevista inicial para una } \\
\text { recolección adecuada de } \\
\text { información. }\end{array}$ & (] \\
\hline $\begin{array}{l}\text { ASEGURAR LA } \\
\text { CORRECTA } \\
\text { IDENTIFICACIÓN DEL } \\
\text { PACIENTE EN LOS } \\
\text { PROCESOS } \\
\text { ASISTENCIALES }\end{array}$ & $\begin{array}{l}\text { Falla de identificación del } \\
\text { paciente neonato. }\end{array}$ & $\begin{array}{l}\text { Diseño de formatos de historia clínica que contengan los datos } \\
\text { personales necesarios e indispensables para lograr una } \\
\text { adecuada identificación. }\end{array}$ & $\begin{array}{l}\text { Auditar los informes de trasladas } \\
\text { con el documento de identificación } \\
\text { del usuario a fin de verificar la } \\
\text { calidad del dato. }\end{array}$ & $\begin{array}{l}\text { El 100\% de los traslados son } \\
\text { auditados para su debida } \\
\text { verificación de la identificación } \\
\text { correcta del usuario. }\end{array}$ & $\begin{array}{l}\text { El } 100 \% \text { de los traslados son } \\
\text { auditados para su debida } \\
\text { verificación de la identificación } \\
\text { correcta del usuario por medio } \\
\text { aplicación de muestras. }\end{array}$ & 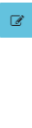 \\
\hline $\begin{array}{l}\text { ASEGURAR LA } \\
\text { CORRECTA } \\
\text { IDENTIFICACIÓN DEL } \\
\text { PACIENTE EN LOS } \\
\text { PROCESOS } \\
\text { ASISTENCIALES }\end{array}$ & $\begin{array}{l}\text { Procesos de captura de } \\
\text { datos incompletos de mala } \\
\text { calidad o equivocados }\end{array}$ & $\begin{array}{l}\text { Contar con los equipos necesarios para la adecuada } \\
\text { recolección de los datos del paciente, (Formatos). }\end{array}$ & $\begin{array}{l}\text { Velar que la información recopilada } \\
\text { en la historia clínica sea la correcta, } \\
\text { validar mediante documento del } \\
\text { usuario la calidad del dato. }\end{array}$ & $\begin{array}{l}\text { El } 100 \% \text { del personal asistencial } \\
\text { asegura la correcta identificación } \\
\text { de los usuarios debido a excelente } \\
\text { calidad en procedimiento de los } \\
\text { datos. }\end{array}$ & $\begin{array}{l}\text { El } 72 \% \text { del personal asistencial } \\
\text { asegura la correcta identificación } \\
\text { de los usuarios debido a excelente } \\
\text { calidad en procedimiento de los } \\
\text { datos. }\end{array}$ & $\square$ \\
\hline $\begin{array}{l}\text { ASEGURAR LA } \\
\text { CORRECTA } \\
\text { IDENTIFICACIÓN DEL } \\
\text { PACIENTE EN LOS } \\
\text { PROCESOS } \\
\text { ASISTENCIALES }\end{array}$ & $\begin{array}{l}\text { Procesos de captura de } \\
\text { datos incompletos de mala } \\
\text { calidad o equivocados }\end{array}$ & $\begin{array}{l}\text { Diseño de formatos de Historia Clínica que contenga lo datos } \\
\text { personales necesarios e indispensables para lograr una } \\
\text { adecuada identificación. }\end{array}$ & $\begin{array}{l}\text { Auditar los informes de trasladas } \\
\text { con el documento de identificación } \\
\text { del usuario a fin de verificar la } \\
\text { calidad del dato. }\end{array}$ & $\begin{array}{l}\text { El } 100 \% \text { de los traslados son } \\
\text { auditados para su debida } \\
\text { verificación de la identificación } \\
\text { correcta del usuario. }\end{array}$ & $\begin{array}{l}\text { El } 100 \% \text { de los traslados son } \\
\text { auditados para su debida } \\
\text { verificación de la identificacion } \\
\text { correcta del usuario por medio } \\
\text { aplicación de muestras. }\end{array}$ & 匹 \\
\hline
\end{tabular}

De acuerdo con la información que muestra la figura 1, en la institución se desarrolla la medición inicial del comportamiento del personal asistencial Frente a estas prácticas, se evidencia, por una parte, que el $28 \%$ del personal asistencial no realiza una adecuada entrevista de salud al paciente al momento del ingreso de este a la institución, por otra parte, el personal asistencial no cuenta con los equipos necesarios para la adecuada recolección de los datos del paciente. Por estas razones se presentó el incremento de los incidentes durante el mes de abril. Con base a estos hallazgos, se desarrolla un plan de acción y de mitigación que se registra en la plataforma (ver figuras 3 a 5). 


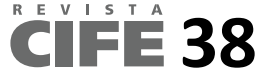

Figura 3. Plan de acción

Editar Plan de Accion : Practica Segura : 184

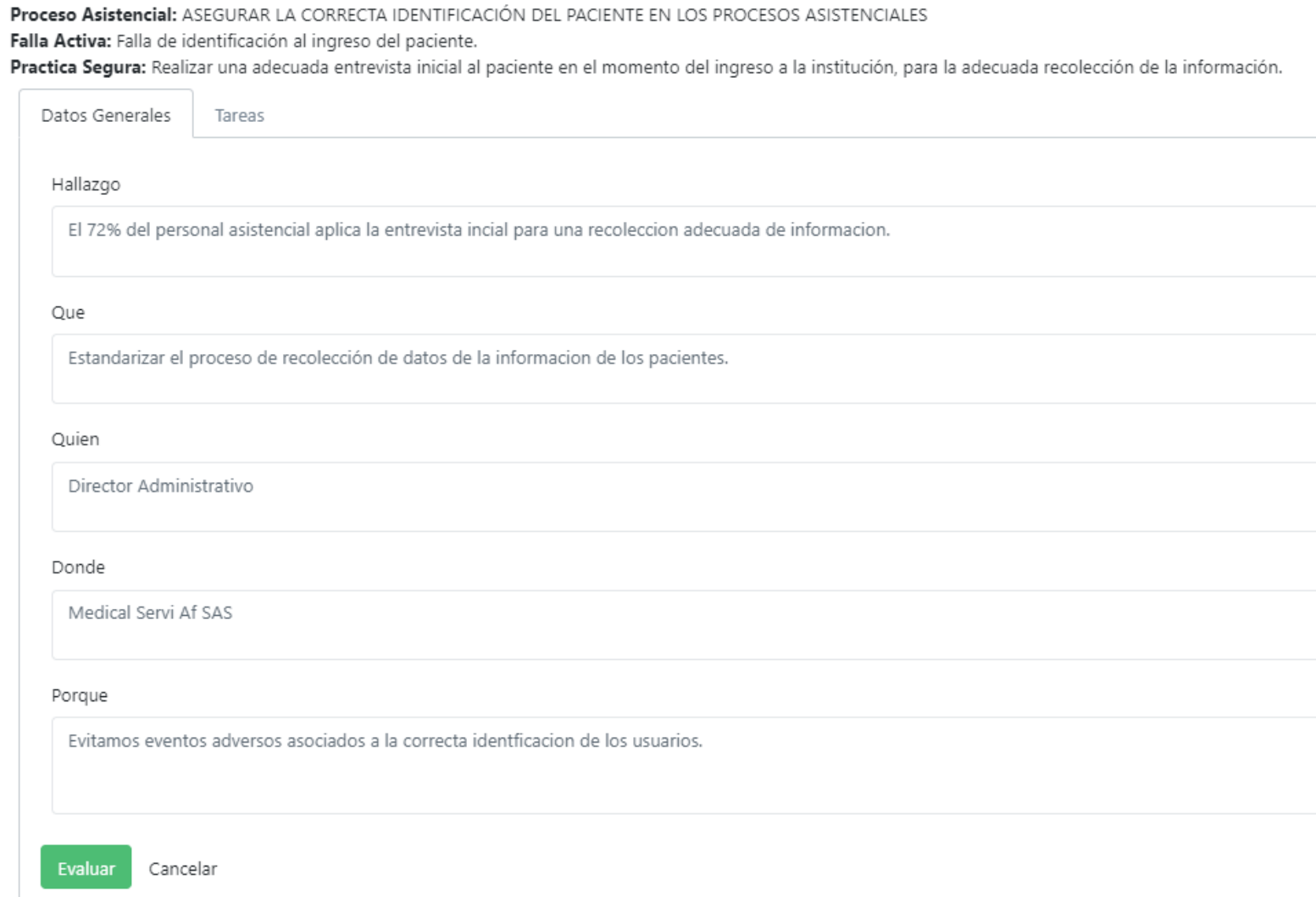

Figura 4. Tareas para la mitigación

Editar Plan de Accion : Practica Segura : 186

Proceso Asistencial: ASEGURAR LA CORRECTA IDENTIFICACIÓN DEL PACIENTE EN LOS PROCESOS ASISTENCIALES

Falla Activa: Procesos de captura de datos incompletos de mala calidad o equivocados

Practica Segura: Diseño de formatos de Historia Clínica que contenga lo datos personales necesarios e indispensables para lograr una adecuada identificación.

Datos Generales Tareas

$+$

Show $10 \vee$ entries

Nombre de la Tarea

Diseñar formatos de historia Clínica que contenga los datos personales necesarios e indispensables para lograr una adecuada identificación del paciente.

Socializar los formatos diseñados de Historia Clínica,que contienen los datos personales necesarios para la adecuada identificación del paciente.

No data available in table

Showing 0 to 0 of 0 entries

\begin{tabular}{|l|l|l|l|}
\hline \multicolumn{5}{|c|}{ Search: } \\
\hline Fecha de Inicio & Fecha de Finalizacion & Estado & \\
\hline 01/08/2019 09:08:00 & $30 / 12 / 2019$ 10:53:00 & En Curso & $\equiv$ \\
\hline 01/08/2019 09:56:00 & $30 / 12 / 2019$ 10:56:00 & En Curso & $\equiv$ @ \\
\hline
\end{tabular}

Durante los periodos comprendidos entre los meses de mayo a diciembre, se cumplió la meta institucional de cero incidentes o eventos asociados a la identificación de los usuarios por medio del seguimiento mensual y el aprendizaje organizacional. 


\section{Ventajas de la aplicación}

La Centralización de la información, obliga al asesor de calidad o de seguridad del paciente a dejar consignadas las evidencias de las acciones desarrolladas en el periodo de tiempo en que estas fueron ejecutadas; en otras palabras, la aplicación garantiza que se continúe con el proceso de seguridad del paciente y de calidad, sin importar quién sea el responsable.

El uso de un panel de control gerencial, dividido en: indicadores, gestión de eventos adversos e incidentes, mejoramiento continuo por medio de las prácticas seguras y programas de vigilancias (hemovigilancia, reactivovigilancia, farmacovigilancia y tecnovigilancia) lo que permite verificar en tiempo real el cumplimiento de las actividades, la gestión de tareas y los cargues de información obligatorios a los entes de control.

La plataforma se encuentra diseñada para que se realice un proceso de planeación — por medio de un cronograma de actividades, la autoevaluación de las prácticas asistenciales seguras y la organización mediante la aplicación de las matrices costo, riesgo, volumen y probabilidad e impacto - y de dirección — por medio de la evaluación del cumplimiento de las tareas y el control a través de los indicadores y el panel de control gerencial sumado a la integración del personal por medio de la asignación de roles y tareas en el panel de control y la matriz $5 \mathrm{~W} 1 \mathrm{H}-$.

El acceso a la información desde cualquier dispositivo electrónico, por medio de un nombre de usuario y su respectiva contraseña, permite la interconectividad entre el personal asistencial y el administrativo. Además, contribuye a la realización del reporte de eventos adversos e incidentes y a la gestión de estos. De este modo, las entidades encargadas del control pueden ver, en tiempo real, el cumplimiento de los programas de seguridad dirigidos a la atención de los pacientes de los entes que cuentan con la herramienta para desarrollar procesos de inspección, vigilancia y control.

Otra ventaja es que se pueden desarrollar las actualizaciones necesarias para mejorar el desempeño de la aplicación. Así con base en los lineamientos normativos, la seguridad del paciente, pues esta tecnología permite que las entidades de control desarrollen periódicamente las actualizaciones a la normatividad que sean necesarias.

\section{Limitaciones}

La principal limitación de la aplicación está asociada al tiempo que se tardaría a integrar al personal en la utilización de la herramienta, especialmente a que se debe contar con unos fundamentos teóricos de base en seguridad de paciente para utilizarla correctamente; sin embargo, luego de que las curvas de aprendizaje se alineen, el proceso sería continuo ya que la herramienta es muy intuitiva. 
La seguridad de la información puede llegar a ser vulnerada por virus tales como el ransomware, si no se toman medidas de seguridad tales como la realización de copias de seguridad de las bases de datos y la utilización de antivirus por parte de las instituciones que utilicen el sistema de información.

\section{Optimización organizacional}

Dentro de la planeación organizacional, específicamente en el análisis del entorno externo, la aplicación está en armonía con el entorno legal colombiano, dentro del sistema obligatorio de la calidad en salud, dando cumplimiento al estándar número 5 de los procesos prioritarios asistenciales del sistema único de habilitación, así como del plan de auditoría para el mejoramiento de la calidad en salud con orientación en seguridad del paciente. Puesto que los lineamientos normativos exigen que dichos procesos sean dinámicos y de mejoramiento continuo, la aplicación permite que la organización evidencie el mejoramiento de la calidad en la prestación de los servicios de salud por medio de la implementación de las prácticas asistenciales seguras.

Tanto la implementación de estas prácticas como el mejoramiento continuo fortalecen el talento humano de la organización, principalmente a la continua capacitación y asignación de responsabilidades asociadas a la seguridad del paciente, pues aseguran la correcta identificación de los usuarios, confirman la funcionabilidad del consentimiento informado, garantizan la administración segura de medicamentos ilustran de manera adecuada al paciente en el autocuidado ayudan a la prevención de úlceras por presión, evitan la frecuencia de caída de los usuarios; entre muchas otras acciones que abordan al usuario desde una perspectiva holística, para garantizar un entorno seguro.

El costo de la baja calidad es muy alto, principalmente por no permitir identificar riesgos que son mitigables. Esta aplicación permite minimizar el riesgo inherente de la prestación de los servicios de salud y llevarlos a un riesgo residual gestionable, que se controla, se evalúa y se mejora continuamente, para garantizar la seguridad en la atención y mejorar los procesos asistenciales, financieros, de talento humano y de servicio al cliente.

\section{Costo de la aplicación}

Es importante aclarar que el propósito de la aplicación es que llegue al mayor número de IPS, profesionales independientes y empresas de transporte especial, la modalidad de asignación de licenciamiento se generaría por medio del arrendamiento mensual de la aplicación y los planes tarifarios variarían de acuerdo al número de usuarios que interactúen con la misma. De igual forma, para establecer el costo por usuario se tendrá en cuenta el salario mínimo diario legal vigente, el cual no podrá exceder el valor de un día de salario por usuario como tarifa máxima, lo que permite modelos de contratación por menor cuantía, es decir, acceder 
a la aplicación sin tener formalidades y/o licitaciones para las Empresas Sociales del Estado; dicha información se aclara en la siguiente tabla:

Tabla 1. Relación de costos

\begin{tabular}{|c|c|c|c|c|c|}
\hline \multicolumn{4}{|c|}{ Número de usuarios } & \multirow{2}{*}{$\begin{array}{c}\text { \% SMDLV } \\
0,35\end{array}$} & \multirow{2}{*}{$\begin{array}{l}\text { Valor mensual } \\
\$ 105336\end{array}$} \\
\hline 1 & $\mathrm{a}$ & 10 & usuarios & & \\
\hline 11 & $\mathrm{a}$ & 20 & usuarios & 0,37 & $\$ 222377$ \\
\hline 25 & $\mathrm{a}$ & 30 & usuarios & 0,4 & $\$ 351121$ \\
\hline 31 & $\mathrm{a}$ & 40 & usuarios & 0,42 & $\$ 491570$ \\
\hline 41 & $\mathrm{a}$ & 50 & usuarios & 0,44 & $\$ 643722$ \\
\hline 51 & $\mathrm{a}$ & 60 & usuarios & 0,46 & $\$ 807579$ \\
\hline 61 & $\mathrm{a}$ & 70 & usuarios & 0,48 & $\$ 983139$ \\
\hline 71 & $\mathrm{a}$ & 80 & usuarios & 0,5 & $\$ 1170404$ \\
\hline 81 & $\mathrm{a}$ & 90 & usuarios & 0,52 & $\$ 1369373$ \\
\hline 91 & $\mathrm{a}$ & 100 & usuarios & 0,54 & $\$ 1580045$ \\
\hline 101 & $\mathrm{a}$ & 200 & usuarios & 0,74 & $\$ 4330495$ \\
\hline 300 & $\mathrm{a}$ & Más & usuarios & 0,95 & $\$ 9414437$ \\
\hline
\end{tabular}

\section{Conclusiones}

Desarrollar un sistema de gestión del conocimiento que mejore los indicadores de gestión de la seguridad del paciente y el análisis de eventos adversos en el desarrollo de las actividades de IPS, profesionales independientes y transporte asistencial, permite contribuir a la política de 'Seguridad del Paciente', que actualmente se encuentra liderada por el Sistema Obligatorio de Garantía de Calidad de la Atención en Salud en Colombia, cuyo objetivo primordial es la reducción y mitigación de la ocurrencia de eventos adversos, que permita tener mejores instituciones, más seguras y altamente competitivas.

Para que este sistema fuera posible, se integró con los indicadores de monitorización del cuadro de mando del Sistema Obligatorio de Garantía de la Calidad -infecciones hospitalarias, complicaciones intra y posoperatorias, eventos centinela, riesgos obstétricos y otros eventos adversos asistenciales mediante un enfoque cuantitativo y cualitativo, donde se permite el análisis de actividades e indicadores de resultados. Cumpliendo de esta manera con los enfoques de medición epidemiológica (incidencia, prevalencia y factores de riesgo 
asociadas) Lo que llevó al diseño de un sistema de notificación que permite analizar, rediseñar, disminuir y contribuir a la gestión de la calidad y seguridad del paciente.

Finalmente, se contribuye al perfeccionamiento de la elaboración de reportes y análisis de eventos adversos, organizando y estandarizando los incidentes que ocurren durante la prestación del servicio, con el fin de priorizarlos, encontrando su causa raíz y su probabilidad de prevalencia.

Este sistema busca inculcar en las instituciones la prioridad de la seguridad del paciente, viéndose como una organización no punible frente a los errores y eventos adversos que se presenten. Al mismo tiempo, se promueve una cultura de aprendizaje organizacional, que involucra a todos los actores y equipos interdisciplinarios que trabajan en el ámbito de la salud. De esta manera, se contribuye a la utilización de un instrumento fiable para el reporte de eventos e incidentes adversos, garantizando la confidencialidad de la información y recordando que esta no constituye un factor sancionatorio.

El sistema de información finaliza con un plan de acción, el cual incluye información de la priorización de los factores contributivos frente al impacto sobre la seguridad futura de los pacientes; una lista de acciones identificada por un análisis estratégico formulado, diseñado y desarrollado; un responsable encargado de implementar las acciones; un tiempo de implementación, posterior a un seguimiento a la ejecución del plan; y, finalmente, un cierre formal, cuando la acción se haya efectuado fijando fecha de seguimiento para evaluar la efectividad del plan de acción.

Con la creación de este reporte extrainstitucional del evento adverso, se dan a conocer las diferentes fallas que presentan las instituciones de salud para que su diagnóstico sirva a todos los actores del sistema de manera estandarizada, obteniendo un informe detallado, producto del análisis de las lecciones aprendidas con base en los eventos informados.

\section{Referencias}

Aitken, M. y Gorokhovich, L. (2012). Advancing the Responsible Applying Levers for Change. The IMS Institute for Healthcare Informatics.

Borges, S., Arroyo, C., Borges, M. y Ferreira, A. (2011). Use and Development of Health Information Systems: The Experience of an Organizational Unit Responsible for the Technological Services at Public Hospitals. JISTEM Journal of Information Systems and Technology Management, 8(1), 155-178. https://doi.org/10.4301/s1807-17752011000100008

Chang, W. (2011). The Nurse Administrator in Transition: Reflections and Future Perspectives. Journal of Nursing, 58(3), 12-16. 
Fatima, A. y Colomo-Palacios, R. (2018). Security Aspects in Healthcare Information Systems: A Systematic Mapping. Procedia Computer Science, 138, 12-19.

Janssen, M. y Behr-Gross, M. (2014). Trends and Observations on the Collection, Testing and Use of Blood and Blood Components in Europe - 2001-2008 Report. http://193.164.228.37/medias/fichiers/trends_and_observations_on_the_collection_t esting_and_use_of_blood_and_blood_components_in_europe.pdf

Jha, A., Larizgoitia, I., Audera-López, C., Prasopa-Plaizier, N., Waters, H. y Bates, D. (2013). The Global Burden of Unsafe Medical Care: Analytic Modelling of Observational Studies. BMJ Quality and Safety, 22(10), 809-815.

Organización Mundial de la Salud. (2002). 55va Asamblea mundial de la salud. Ginebra, 13-18 de mayo de 2002. Resoluciones y decisiones. Anexos. https://apps.who.int/iris/bitstream/handle/10665/258955/WHA55-2002-REC-1spa.pdf? sequence $=1 \&$ isAllowed $=y$

Raskob, G., Angchaisuksiri, P., Blanco, A., Buller, H., Gallus, A., Hunt, B., Hylek, E., Kakkar, A., Konstantinides, S., McCumber, M., Ozaki, Y., Wendelboe, A. y Weitz, J. (2014). Thrombosis: A Major Contributor to Global Disease Burden. ISTH Steering Committee for World Thrombosis Day. Thrombosis Research, 12(10), 1580-1590. https://doi.org/10.1016/j.thromres.2014.08.014

Singh, H., Meyer, A. y Thomas, E. (2014). The Frequency of Diagnostic Errors in Outpatient Care: Estimations from Three Large Observational Studies Involving US Adult Populations. BMJ Quality and Safety, 23(9), 727-731. https://doi.org/10.1136/bmjqs-2013-002627

Summers, A. (2006). Administración de la calidad. Pearson Educación.

Wei, X. (2011). Hospital Information System Management and Security Maintenance. Communications in Computer and Information Science, 234, 418-421. https://doi.org/10.1007/978-3-642-24091-1_54

World Health Organization. (2005). Reto mundial en pro de la seguridad del paciente 2005-2006. https://www.who.int/patientsafety/information_centre/GPSC_Launch_sp.pdf

World Health Organization. (2011). Report on the Burden of Endemic Health Care-Associated Infection Worldwide. Clean Care is Safer Care. https://apps.who.int/iris/bitstream/handle/10665/80135/9789241501507_eng.pdf?se quence $=1$ 


\section{Bibliografía consultada}

Altés, J. (2013). Papel de las tecnologías de la información y la comunicación en la medicina actual. Seminarios de la Fundación Española de Reumatología, 14(2), 31-35.

Fernández, S. (2015). Los eventos adversos y la seguridad del paciente. http://www.conamed.gob.mx/gobmx/boletin/pdf/boletin3/eventos_adversos.pdf

Jedamzik, S. (2019). Digital Health and Nursing: The Future is Now. Unfallchirurg, 122(9), 670-675

Kohno, R., Kobayashi, T., Sugimoto, C., Kinjo, Y., Hämäläinen, M. y Iinatti, J. (2019). Medical Healthcare Network Platform and Big Data Analysis Based on Integrated ICT and Data Science with Regulatory Science. IEICE Transactions on Communications, E102B(6), 1078-1087.

Ministerio de Salud y Protección Social. (2015). Evaluación de la frecuencia de eventos adversos y monitoreo de aspectos claves relacionados con la seguridad del paciente. Paquetes Instruccionales: Guía Técnica "Buenas prácticas para la seguridad del paciente en la atención en salud" Versión 2.0. Minsalud. https://doi.org/https://doi.org/10.1016/j.foreco.2016.02.016

Organización Panamericana de la Salud. (2014). Conversaciones sobre eSalud. Gestión de información, diálogos e intercambio de conocimiento para acercarnos al acceso universal a la salud (Issue January).

https://iris.paho.org/bitstream/handle/10665.2/28391/9789275318287_spa.pdf?sequ ence $=1 \&$ isAllowed $=\mathrm{y}$

Vallejo-Gutiérrez, P., Bañeres-Amella, J., Sierra, E., Casal, J. y Agra, Y. (2014). Lessons Learnt from the Development of the Patient Safety Incidents Reporting and Learning System for the Spanish National Health System: SiNASP. Revista de Calidad Asistencial, 29(2), 69-77. https://doi.org/10.1016/j.cali.2013.09.007 


\section{Anexo técnico}

\section{Certificado de registro de soporte lógico (software)}

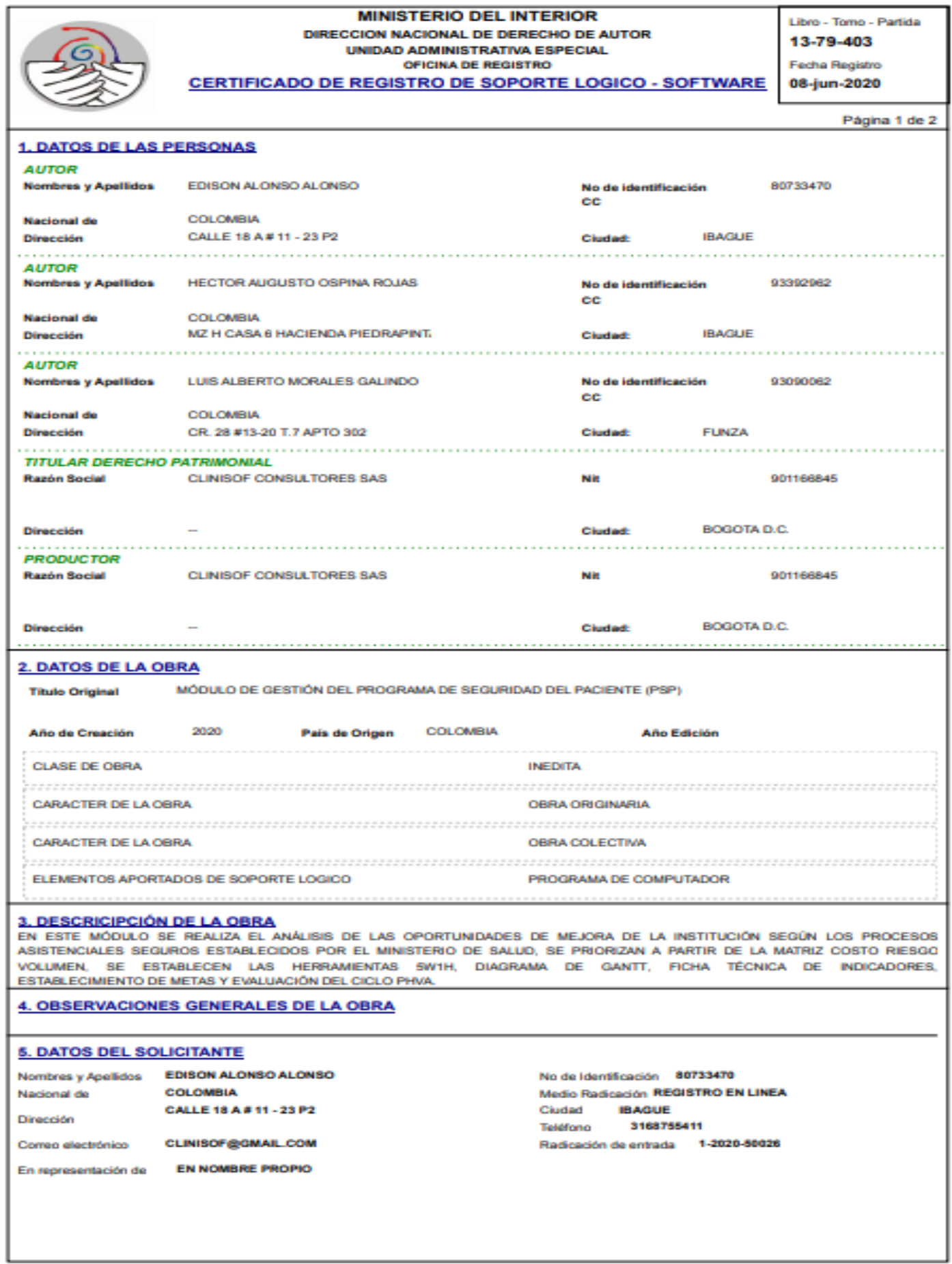




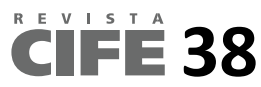

\begin{tabular}{|c|c|c|}
\hline & $\begin{array}{c}\text { MINISTERIO DEL INTERIOR } \\
\text { DIRECCION NACIONAL DE DERECHO DE AUTOR } \\
\text { UNIDAD ADMINISTRATNA ESPECIAL } \\
\text { OFICINA DE REQISTRO } \\
\text { CERTIFICADO DERECISTRO DE SOPORTELOGICO - SOFTWARE }\end{array}$ & $\begin{array}{l}\text { Libro- Tamo - Pantia } \\
\text { 13-79-403 } \\
\text { Fecha Alegietro } \\
08-j u n-2020\end{array}$ \\
\hline & & Pagina 2 de 2 \\
\hline & $\begin{array}{l}\text { MANUEL ANTONIO MORA CUELLAR } \\
\text { JEFE OFICINA DE REGISTRO }\end{array}$ & \\
\hline$m z p$ & & \\
\hline
\end{tabular}

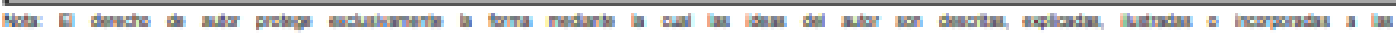

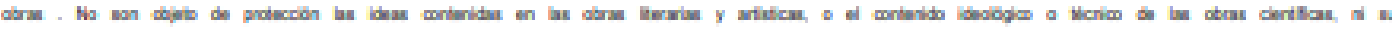

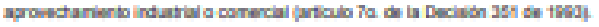

$$
\text { (c) (1) () }
$$

